\title{
A NEW NUMERICAL TECHNIQUE FOR SOLVING FRACTIONAL BRATU'S INITIAL VALUE PROBLEMS IN THE CAPUTO AND CAPUTO-FABRIZIO SENSE
}

\author{
Ali Khalouta, Abdelouahab Kadem \\ Laboratory of Fundamental and Numerical Mathematics \\ Departement of Mathematics, Faculty of Sciences \\ Ferhat Abbas Sétif University 1, 19000 Sétif, Algeria \\ nadjibkh@yahoo.fr,abdelouahabk@yahoo.fr
}

Received: 7 January 2020; Accepted: 18 February 2020

\begin{abstract}
The purpose of this paper is to propose a new numerical technique called the natural decomposition method (NDM) for solving fractional Bratu's initial value problems (FBIVP) in the Caputo and Caputo-Fabrizio sense. The NDM is a combined form of the natural transform method and the Adomian decomposition method. The numerical example is provided in order to validate the efficiency and reliability of the proposed method. The obtained results reveal that the proposed method is a very efficient and simple tool for solving fractional differential equations.
\end{abstract}

MSC 2010: $34 A 08,26 A 33,34 K 28,35 C 10$

Keywords: fractional Bratu's initial value problem, Caputo fractional derivative, Caputo-Fabrizio fractional derivative, natural transform method, Adomian decomposition method

\section{Introduction}

It is well known that the classical Bratu-type equation is of the form

$$
u^{\prime \prime}(t)+\lambda \exp (u(t))=0,0<t<1 .
$$

The exact solution is [1]

$$
u(t)=-2 \ln \left[\frac{\cosh \left(\left(t-\frac{1}{2}\right) \frac{\theta}{2}\right)}{\cosh \left(\frac{\theta}{4}\right)}\right],
$$

where $\theta$ satisfies

$$
\theta=\sqrt{2 \lambda} \cosh \left(\frac{\theta}{4}\right) .
$$


The problem has zero, one or two solutions when $\lambda>\lambda_{c}, \lambda=\lambda_{c}$, and $\lambda<\lambda_{c}$, respectively, where the critical value $\lambda_{c}$ satisfies the equation

$$
1=\frac{1}{4} \sqrt{2 \lambda_{c}} \sinh \left(\frac{\theta_{c}}{4}\right)
$$

where

$$
\lambda_{c}=3.513830719 \text {. }
$$

The classical Bratu-type equation was used in a large variety of applications such as the fuel ignition model of the thermal combustion theory, the model of the thermal reaction process, the Chandrasekhar model of the expansion of the universe, questions in geometry and relativity about the Chandrasekhar model, chemical reaction theory, radiative heat transfer and nanotechnology [1-4].

Several authors have proposed various methods to obtain the approximate solution of the classical Bratu-type equation such as: the Adomian decomposition method (ADM) [4], variational iteration method (VIM) [5], homotopy perturbation method (HPM) [6], optimal homotopy analysis method (OHAM) [7], Optimal perturbation iteration method (OPIM) [8], Lie-group shooting method (LGSM) [9].

The main objective of this paper is to propose a powerful method called the natural decomposition method (NDM) for solving the fractional Bratu's initial value problems (FBIVP) with two different fractional derivative operators in the forms

$$
\left\{\begin{array}{l}
D^{\alpha} u(t)+\lambda \exp (u(t))=0 \\
u(0)=a_{0}, u^{\prime}(0)=a_{1}
\end{array}\right.
$$

where $D^{\alpha}$ is the Caputo fractional derivative operator of order $\alpha, 1<\alpha \leq 2,0<t<1$ and $\lambda \in \mathbb{R}$, and

$$
\left\{\begin{array}{c}
\mathscr{D}^{(\mu)} u(t)+\lambda \exp (u(t))=0, \\
u(0)=a_{0}, u^{\prime}(0)=a_{1},
\end{array}\right.
$$

where $\mathscr{D}^{(\mu)}=\mathscr{D}^{(\alpha+1)}$ is the Caputo-Fabrizio fractional derivative operator of order $\mu=\alpha+1,0<\alpha \leq 1$.

In recent years, many numerical and approximate analytical methods have been developed for solving fractional differential equations in the Caputo and Caputo-Fabrizio sense. For more details, see [10-18].

\section{Preliminaries and notations}

In this section, we present the main definitions and properties of the fractional calculus theory and natural transform. 
Definition 1 [19] Let $u \in L^{1}(0,1)$. The left sided Riemann-Liouville fractional integral of order $\alpha \geq 0$ is defined by

$$
I^{\alpha} u(t)= \begin{cases}\frac{1}{\Gamma(\alpha)} \int_{0}^{t}(t-\tau)^{\alpha-1} u(\tau) d \tau, & \alpha>0 \\ u(t), & \alpha=0\end{cases}
$$

where $\Gamma($.$) is the Euler gamma function.$

Definition 2 [19] Let $u^{(n)} \in L^{1}(0,1)$. The left sided Caputo fractional derivative of order $\alpha \geq 0$ is defined by

$$
D^{\alpha} u(t)=\left\{\begin{array}{lc}
\frac{1}{\Gamma(n-\alpha)} \int_{0}^{t}(t-\tau)^{n-\alpha-1} u^{(n)}(\tau) d \tau, & n-1<\alpha<n, \\
u^{(n)}(t), & \alpha=n,
\end{array}\right.
$$

where $n=[\alpha]+1$ with $[\alpha]$ being the integer part of $\alpha$.

In equation (9) if transformations happen as follows

$$
(t-\tau)^{n-\alpha-1} \longrightarrow \exp \left[-\frac{\alpha(t-\tau)}{1-\alpha}\right] \text { and } \frac{1}{\Gamma(n-\alpha)} \longrightarrow \frac{M(\alpha)}{1-\alpha},
$$

the new definition of the fractional operator is expressed by Caputo and Fabrizio.

Definition 3 [20] Let $u \in H^{1}(0,1)$, then the Caputo-Fabrizio fractional derivative of order $\alpha, 0<\alpha \leq 1$ is defined as

$$
\mathscr{D}^{(\alpha)} u(t)=\frac{M(\alpha)}{1-\alpha} \int_{0}^{t} u^{\prime}(\tau) \exp \left[-\frac{\alpha(t-\tau)}{1-\alpha}\right] d \tau,
$$

where $M(\alpha)$ is a normalization function that satisfies $M(0)=M(1)=1$.

From equation (11) it follows that if $u(t)=C$ is a constant, then $\mathscr{D}^{(\alpha)} C=0$ as in the sense of Caputo [19].

If $u \notin H^{1}(0,1)$, then its fractional derivative is redefined as [20]

$$
\mathscr{D}^{(\alpha)} u(t)=\frac{\alpha M(\alpha)}{1-\alpha} \int_{0}^{t}(u(t)-u(\tau)) \exp \left[-\frac{\alpha(t-\tau)}{1-\alpha}\right] d \tau, 0<t<1 .
$$

For $n \geq 1$ and $0<\alpha \leq 1$, the fractional derivative of order $(\alpha+n)$ is defined by

$$
\mathscr{D}^{(\alpha+n)} u(t)=\mathscr{D}^{(\alpha)}\left(\mathscr{D}^{(n)} u(t)\right) .
$$


The above Caputo-Fabrizio fractional derivative was later modified by Jorge Losada and Juan José Nieto [21] as

$$
\mathscr{D}^{(\alpha)} u(t)=\frac{(2-\alpha) M(\alpha)}{2(1-\alpha)} \int_{0}^{t} u^{\prime}(\tau) \exp \left[-\frac{\alpha(t-\tau)}{1-\alpha}\right] d \tau, 0<t<1 .
$$

The fractional integral corresponding to the derivative in equation (14) was defined by Jorge Losada and Juan José Nieto in 2015, as follows.

Definition 4 [21] Let $0<\alpha \leq 1$. The fractional integral of order $\alpha$ of $u$ is defined by

$$
\mathscr{I}^{(\alpha)} u(t)=\frac{2(1-\alpha)}{(2-\alpha) M(\alpha)} u(t)+\frac{2 \alpha}{(2-\alpha) M(\alpha)} \int_{0}^{t} u(\tau) d \tau, 0<t<1
$$

From the definition in equation (15), the fractional integral of the Caputo-Fabrizio type of a function $u$ of order $0<\alpha \leq 1$ is an average between function $u$ and its one order integral, i.e.,

$$
\frac{2(1-\alpha)}{(2-\alpha) M(\alpha)}+\frac{2 \alpha}{(2-\alpha) M(\alpha)}=1
$$

Therefore,

$$
M(\alpha)=\frac{2}{2-\alpha}, 0<\alpha \leq 1 .
$$

Due to this, Losada and Nieto remarked that the Caputo-Fabrizio fractional derivative can redefined as

Definition 5 [21] Let $0<\alpha \leq 1$. The fractional Caputo-Fabrizio derivative of order $\alpha$ of a function $u$ is given by

$$
\mathscr{D}^{(\alpha)} u(t)=\frac{1}{1-\alpha} \int_{0}^{t} u^{\prime}(\tau) \exp \left[-\frac{\alpha(t-\tau)}{1-\alpha}\right] d \tau, 0<t<1 .
$$

Definition 6 [22] The natural transform is defined over the set of functions

$$
A=\left\{u(t) / \exists M, \xi_{1}, \xi_{2}>0,|u(t)|<M \exp \left(\frac{|t|}{\xi_{j}}\right), \text { if } t \in(-1)^{j} \times[0, \infty)\right\},
$$

by the following integral

$$
\mathscr{N}^{+}[u(t)]=R^{+}(s, v)=\frac{1}{v} \int_{0}^{+\infty} \exp \left(-\frac{s t}{v}\right) u(t) d t, s, v \in(0, \infty) .
$$


Some basic properties of the natural transform are given as follows

Property 1: The natural transform is a linear operator

$$
\mathscr{N}^{+}[a u(t) \pm b w(t)]=a \mathscr{N}^{+}[u(t)] \pm b \mathscr{N}^{+}[w(t)], a, b \in \mathbb{R} .
$$

Property 2: If $u^{(n)}(t)$ is the $n$-th derivative of function $u(t)$ with respect to the time variable $t$, then its natural transform is given by

$$
\mathscr{N}^{+}\left[u^{(n)}(t)\right]=R_{n}^{+}(s, v)=\frac{s^{n}}{v^{n}} R^{+}(s, v)-\sum_{k=0}^{n-1} \frac{s^{n-(k+1)}}{v^{n-k}} u^{(k)}(0) .
$$

Property 3: (Convolution property) Suppose $U^{+}(s, v)$ and $W^{+}(s, v)$ are the natural transforms of $u(t)$ and $w(t)$, respectively, both defined in the set $A$. Then the natural transform of their convolution is given by

$$
\mathscr{N}^{+}[(u * w)(t)]=v U^{+}(s, v) W^{+}(s, v) .
$$

Property 4: Some special natural transforms

$$
\mathscr{N}^{+}[1]=\frac{1}{s}, \mathscr{N}^{+}[t]=\frac{v}{s^{2}}, \mathscr{N}^{+}\left[\frac{t^{n}}{n !}\right]=\frac{v^{n}}{s^{n+1}}, n=0,1,2, \ldots
$$

Property 5: If $\alpha>-1$, then the natural transform of $t^{\alpha}$ is given by

$$
\mathscr{N}^{+}\left[t^{\alpha}\right]=\Gamma(\alpha+1) \frac{v^{\alpha}}{s^{\alpha+1}} .
$$

Theorem 1 [23] Let $n \in \mathbb{N}^{*}$ and $\alpha>0$ be such that $n-1<\alpha \leq n$ and $R^{+}(s, v)$ be the natural transform of the function $u(t)$, then the natural transform denoted by $R_{\alpha}^{+}(s, v)$ of the Caputo fractional derivative of the function $u(t)$ of order $\alpha$, is given by

$$
\mathscr{N}^{+}\left[D^{\alpha} u(t)\right]=R_{\alpha}^{+}(s, v)=\frac{s^{\alpha}}{v^{\alpha}} R^{+}(s, v)-\sum_{k=0}^{n-1} \frac{s^{\alpha-(k+1)}}{u^{\alpha-k}} u^{(k)}(0) .
$$

Theorem 2 The natural transform of the Caputo-Fabrizio fractional derivative of the function $u(t)$ of order $\alpha+n$, where $0<\alpha \leq 1$ and $n \in \mathbb{N} \cup\{0\}$, is given by

$$
\mathscr{N}^{+}\left[\mathscr{D}^{(\alpha+n)} u(t)\right]=\frac{1}{s-\alpha(s-v)}\left[\frac{s^{n+1}}{v^{n}} \mathscr{N}^{+}(u(t))-\sum_{k=0}^{n}\left(\frac{s}{v}\right)^{n-k} u^{(k)}(0)\right] .
$$

Proof By the Definition of the Caputo-Fabrizio fractional derivative 5 and using the relation (13), we have 


$$
\begin{aligned}
\mathscr{N}^{+}\left[\mathscr{D}^{(\alpha+n)} u(t)\right] & =\mathscr{N}^{+}\left[\mathscr{D}^{(\alpha)}\left(\mathscr{D}^{(n)} u(t)\right)\right] \\
& =\frac{1}{1-\alpha} \frac{1}{v} \int_{0}^{+\infty} \exp \left(-\frac{s t}{v}\right) \int_{0}^{t} u^{(n)}(\tau) \exp \left[-\frac{\alpha(t-\tau)}{1-\alpha}\right] d \tau \\
& =\frac{1}{1-\alpha} \frac{1}{v} \int_{0}^{+\infty} \exp \left(-\frac{s t}{v}\right)\left(u^{(n)}(t) * \exp \left[-\frac{\alpha t}{1-\alpha}\right]\right) \\
& =\frac{1}{1-\alpha} \mathscr{N}^{+}\left(u^{(n)}(t) * \exp \left[-\frac{\alpha t}{1-\alpha}\right]\right) .
\end{aligned}
$$

Hence, from the Properties 2 and 3 of the natural transform, we have

$$
\begin{aligned}
\mathscr{N}^{+}\left[\mathscr{D}^{(\alpha+n)} u(t)\right] & =\frac{1}{1-\alpha} v \mathscr{N}^{+}\left(u^{(n)}(t)\right) \mathscr{N}^{+}\left(\exp \left[-\frac{\alpha t}{1-\alpha}\right]\right) \\
& =\frac{v}{s-\alpha(s-v)}\left[\frac{s^{n}}{v^{n}} \mathscr{N}^{+}(u(t))-\sum_{k=0}^{n-1} \frac{s^{n-(k+1)}}{v^{n-k}} u^{(k)}(0)\right] \\
& =\frac{v}{s-\alpha(s-v)}\left[\frac{s^{n+1}}{v^{n+1}} \mathscr{N}^{+}(u(t))-\sum_{k=0}^{n} \frac{s^{n-k}}{v^{n-k}} u^{(k)}(0)\right] \\
& =\frac{1}{s-\alpha(s-v)}\left[\frac{s^{n+1}}{v^{n}} \mathscr{N}^{+}(u(t))-\sum_{k=0}^{n}\left(\frac{s}{v}\right)^{n-k} u^{(k)}(0)\right] .
\end{aligned}
$$

\section{Description of the natural decomposition method (NDM)}

Theorem 3 Consider the following FBIVP (6) and (7). Then, by NDM the solution of (6) and (7) is given in the form of infinite series as follows

$$
u(t)=\sum_{n=0}^{\infty} u_{n}(t)
$$

PROOF Consider the following FBIVP (6) and (7). First, we define the nonlinear operator

$$
R u(t)=\exp (u(t)) .
$$

\section{1) For the Caputo fractional derivative}

Equation (6) is written in the form

$$
D^{\alpha} u(t)+\lambda R u(t)=0,0<t<1, \lambda \in \mathbb{R} .
$$


Applying the natural transform on both sides of (30) and using the Theorem 1, we get

$$
\mathscr{N}^{+}[u(t)]=\frac{1}{s} u(0)+\frac{v}{s^{2}} u^{\prime}(0)-\frac{v^{\alpha}}{s^{\alpha}} \mathscr{N}^{+}[\lambda R u(t)] .
$$

After that, let us take the inverse natural transform on both sides of (31), we have

$$
u(t)=a_{0}+a_{1} t-\lambda \mathscr{N}^{-1}\left(\frac{v^{\alpha}}{s^{\alpha}} \mathscr{N}^{+}[R u(t)]\right) .
$$

Now, we represent the solution in an infinite series form

$$
u(t)=\sum_{n=0}^{\infty} u_{n}(t)
$$

and the nonlinear terms $R u(t)$ can be decomposed as

$$
R u(t)=\sum_{n=0}^{\infty} A_{n}(t),
$$

where $A_{n}$ are the Adomian polynomials [24] of $u_{0}, u_{1}, u_{2}, . ., u_{n}$, and it can be calculated by formula given below

$$
A_{n}=\frac{1}{n !} \frac{d^{n}}{d \lambda^{n}}\left[R\left(\sum_{i=0}^{\infty} \lambda^{i} u_{i}\right)\right]_{\lambda=0}, n=0,1,2, \ldots
$$

Using equations (33) and (34), we can rewrite equation (32) as

$$
\sum_{n=0}^{\infty} u_{n}(t)=a_{0}+a_{1} t-\lambda \mathscr{N}^{-1}\left(\frac{v^{\alpha}}{s^{\alpha}} \mathscr{N}^{+}\left[\sum_{n=0}^{\infty} A_{n}(t)\right]\right) .
$$

By comparing both sides of equation (35), we have the following relation

$$
\begin{aligned}
& u_{0}(t)=a_{0}+a_{1} t \\
& u_{1}(t)=-\lambda \mathscr{N}^{-1}\left(\frac{v^{\alpha}}{s^{\alpha}} \mathscr{N}^{+}\left[A_{0}(t)\right]\right), \\
& u_{2}(t)=-\lambda \mathscr{N}^{-1}\left(\frac{v^{\alpha}}{s^{\alpha}} \mathscr{N}^{+}\left[A_{1}(t)\right]\right),
\end{aligned}
$$

In general, the recursive relation is given by

$$
\begin{aligned}
u_{0}(t) & =a_{0}+a_{1} t, \\
u_{n+1}(t) & =-\lambda \mathscr{N}^{-1}\left(\frac{v^{\alpha}}{s^{\alpha}} \mathscr{N}^{+}\left[A_{n}(t)\right]\right), n \geq 0 .
\end{aligned}
$$


Then, the solution of FBIVP (6) is given in the form of infinite series as follows

$$
u(t)=\sum_{n=0}^{\infty} u_{n}(t)
$$

\section{2) For the Caputo-Fabrizio fractional derivative}

Equation (7) is written in the form

$$
\mathscr{D}^{(\alpha+1)} u(t)+\lambda R u(t)=0,0<t<1, \lambda \in \mathbb{R} .
$$

Applying the natural transform on both sides of (39) and using the Theorem 2, we get

$$
\mathscr{N}^{+}[u(t)]=\frac{1}{s} u(0)+\frac{v}{s^{2}} u^{\prime}(0)-\frac{v(s-\alpha(s-v))}{s^{2}} \mathscr{N}^{+}[\lambda R u(t)] .
$$

After that, let us take the inverse natural transform on both sides of (40), we have

$$
u(t)=a_{0}+a_{1} t-\lambda \mathscr{N}^{-1}\left(\frac{v(s-\alpha(s-v))}{s^{2}} \mathscr{N}^{+}[R u(t)]\right) .
$$

Now, we represent the solution in an infinite series form

$$
u(t)=\sum_{n=0}^{\infty} u_{n}(t)
$$

and the nonlinear terms $R u(t)$ can be decomposed as

$$
R u(t)=\sum_{n=0}^{\infty} A_{n}(t) .
$$

Using equations (42) and (43), we can rewrite equation (41) as

$$
\sum_{n=0}^{\infty} u_{n}(t)=a_{0}+a_{1} t-\lambda \mathscr{N}^{-1}\left(\frac{v(s-\alpha(s-v))}{s^{2}} \mathscr{N}^{+}\left[\sum_{n=0}^{\infty} A_{n}(t)\right]\right) .
$$

By comparing both sides of equation (44), we have the following relation

$$
\begin{aligned}
& u_{0}(t)=a_{0}+a_{1} t \\
& u_{1}(t)=-\lambda \mathscr{N}^{-1}\left(\frac{v(s-\alpha(s-v))}{s^{2}} \mathscr{N}^{+}\left[A_{0}(t)\right]\right), \\
& u_{2}(t)=-\lambda \mathscr{N}^{-1}\left(\frac{v(s-\alpha(s-v))}{s^{2}} \mathscr{N}^{+}\left[A_{1}(t)\right]\right),
\end{aligned}
$$


In general the recursive relation is given by

$$
\begin{aligned}
u_{0}(t) & =a_{0}+a_{1} t \\
u_{n+1}(t) & =-\lambda \mathscr{N}^{-1}\left(\frac{v(s-\alpha(s-v))}{s^{2}} \mathscr{N}^{+}\left[A_{n}(t)\right]\right), n \geq 0 .
\end{aligned}
$$

Then, the solution of FBIVP (7) is given in the form of infinite series as follows

$$
u(t)=\sum_{n=0}^{\infty} u_{n}(t)
$$

Theorem 4 Let $\mathscr{B}$ be a Banach space. Then the series solution of the FBIVP (6) and (7) converges to $S \in \mathscr{B}$, if there exists $\gamma, 0<\gamma<1$ such that

$$
\left\|u_{n}\right\| \leq \gamma\left\|u_{n-1}\right\|, \forall n \in \mathbb{N} .
$$

Proof Define the sequences $\left\{S_{n}\right\}_{n \geq 1}$ of partial sums of the series given by the recursive relation (37) or (45) as

$$
S_{n}(t)=u_{1}(t)+u_{2}(t)+u_{3}(t)+\ldots+u_{n}(t),
$$

and we need to show that $\left\{S_{n}\right\}_{n \geq 1}$ are a Cauchy sequences in Banach space $\mathscr{B}$. For this purpose, we consider

$$
\left\|S_{n+1}-S_{n}\right\| \leq\left\|u_{n+1}\right\| \leq \gamma\left\|u_{n}\right\| \leq \gamma^{2}\left\|u_{n-1}\right\| \leq \ldots \leq \gamma^{n+1}\left\|u_{0}\right\| .
$$

For every $n, m \in \mathbb{N}, n \geq m$, by using (47) and triangle inequality successively, we have

$$
\begin{aligned}
\left\|S_{n}-S_{m}\right\| & =\left\|S_{m+1}-S_{m}+S_{m+2}-S_{m+1}+\ldots+S_{n}-S_{n-1}\right\| \\
& \leq\left\|S_{m+1}-S_{m}\right\|+\left\|S_{m+2}-S_{m+1}\right\|+\ldots+\left\|S_{n}-S_{n-1}\right\| \\
& \leq \gamma^{m+1}\left\|u_{0}\right\|+\gamma^{m+2}\left\|u_{0}\right\|+\ldots+\gamma^{n}\left\|u_{0}\right\| \\
& =\gamma^{m+1}\left(1+\gamma+\ldots+\gamma^{n-m-1}\right)\left\|u_{0}\right\| \leq \gamma^{m+1}\left(\frac{1-\gamma^{n-m}}{1-\gamma}\right)\left\|u_{0}\right\| .
\end{aligned}
$$

Since $0<\gamma<1$, so $1-\gamma^{n-m} \leq 1$ then

$$
\left\|S_{n}-S_{m}\right\| \leq \frac{\gamma^{m+1}}{1-\gamma}\left\|u_{0}\right\|
$$

Since $u_{0}$ is bounded, then

$$
\lim _{n, m \longrightarrow \infty}\left\|S_{n}-S_{m}\right\|=0 .
$$

Therefore, the sequences $\left\{S_{n}\right\}_{n \geq 1}$ are Cauchy sequences in Banach space $\mathscr{B}$, so the series solution defined in (28) converges. 
Example 1. Consider the following fractional Bratu's initial value problem with Caputo fractional derivative

$$
\left\{\begin{array}{lr}
D^{\alpha} u(t)-2 \exp (u(t))=0,0<t<1 \\
u(0)=u^{\prime}(0)=0, & 1<\alpha \leq 2 .
\end{array}\right.
$$

For $\alpha=2$, the exact solution of BIVP (52), is (see [25])

$$
u(t)=-2 \ln (\cos t) .
$$

Following the description of the NDM presented in Section 3, gives

$$
\sum_{n=0}^{\infty} u_{n}(t)=2 \mathscr{N}^{-1}\left(\frac{v^{\alpha}}{s^{\alpha}} \mathscr{N}^{+}\left[\sum_{n=0}^{\infty} A_{n}(t)\right]\right),
$$

where $\exp (u(t))=\sum_{n=0}^{\infty} A_{n}(t)$ are the Adomian polynomials that represents the nonlinear term, and it can be calculated by formula (35). For the convenience of the reader, the first few nonlinear terms are as follows:

$$
\begin{aligned}
& A_{0}(t)=\exp \left(u_{0}(t)\right) \\
& A_{1}(t)=u_{1}(t) \exp \left(u_{0}(t)\right), \\
& A_{2}(t)=\left(u_{2}(t)+\frac{1}{2} u_{1}^{2}(t)\right) \exp \left(u_{0}(t)\right), \\
& A_{3}(t)=\left(u_{3}(t)+u_{1}(t) u_{3}(t)+\frac{1}{6} u_{1}^{3}(t)\right) \exp \left(u_{0}(t)\right),
\end{aligned}
$$

From the relationship in (37) and Property 5, we have

$$
\begin{aligned}
& u_{0}(t)=0 \\
& u_{1}(t)=\frac{2}{\Gamma(\alpha+1)} t^{\alpha} \\
& u_{2}(t)=\frac{4}{\Gamma(2 \alpha+1)} t^{2 \alpha} \\
& u_{3}(t)=\frac{4\left[2 \Gamma^{2}(\alpha+1)+\Gamma(2 \alpha+1)\right]}{\Gamma(3 \alpha+1) \Gamma^{2}(\alpha+1)} t^{3 \alpha}
\end{aligned}
$$

Therefore, the approximate solution of FBIVP (52), is given by

$$
u(t)=\frac{2}{\Gamma(\alpha+1)} t^{\alpha}+\frac{4}{\Gamma(2 \alpha+1)} t^{2 \alpha}+\frac{4\left[2 \Gamma^{2}(\alpha+1)+\Gamma(2 \alpha+1)\right]}{\Gamma(3 \alpha+1) \Gamma^{2}(\alpha+1)} t^{3 \alpha}+\ldots
$$


Now, we consider the following fractional Bratu's initial value problem with Caputo-Fabrizio fractional derivative

$$
\left\{\begin{array}{c}
\mathscr{D}^{(\mu)} u(t)-2 \exp (u(t))=0,0<t<1 \\
u(0)=u^{\prime}(0)=0, \mu=\alpha+1,0<\alpha \leq 1 .
\end{array}\right.
$$

For $\mu=2(\alpha=1)$, the exact solution of BIVP (55), is (see [25])

$$
u(t)=-2 \ln (\cos t) .
$$

Following the description of the NDM presented in Section 3, gives

$$
\sum_{n=0}^{\infty} u_{n}(t)=2 \mathscr{N}^{-1}\left(\frac{v(s-\alpha(s-v))}{s^{2}} \mathscr{N}^{+}\left[\sum_{n=0}^{\infty} A_{n}(t)\right]\right) .
$$

Similarly above, and from the relationship in (45) and Property 4, we have

$$
\begin{aligned}
& u_{0}(t)=0 \\
& u_{1}(t)=2(1-\alpha) t+\alpha t^{2} \\
& u_{2}(t)=2(1-\alpha)^{2} t^{2}+\frac{4 \alpha(1-\alpha)}{3} t^{3}+\frac{\alpha^{2}}{6} t^{4} \\
& u_{3}(t)=\frac{8}{3}(1-\alpha)^{3} t^{3}+\frac{7}{3} \alpha(1-\alpha)^{2} t^{4}+\frac{3}{5} \alpha^{2}(1-\alpha) t^{5}+\frac{2}{45} \alpha^{3} t^{6}
\end{aligned}
$$

Therefore, the approximate solution of FBIVP (55), is given by

$$
u(t)=\sum_{n=0}^{\infty} u_{n}(t)=u_{0}(t)+u_{1}(t)+u_{2}(t)+u_{3}(t)+\ldots
$$

Table 1. Numerical comparison between the 4-term approximate solutions by NDM Caputo at $\alpha=2$, NDM Caputo-Fabrizio at $\alpha=1$ and exact solution

\begin{tabular}{|c|c|c|c|c|c|}
\hline$t$ & $u_{N D M-C}$ & $u_{N D M-C F}$ & Exact solution & $\left|u_{\text {exact }}-u_{N D M-C}\right|$ & $\left|u_{\text {exact }}-u_{N D M-C F}\right|$ \\
\hline 0.1 & 0.010017 & 0.010017 & 0.010017 & $1.3536 \times 10^{-10}$ & $1.3536 \times 10^{-10}$ \\
0.2 & 0.040270 & 0.040270 & 0.040270 & $3.4994 \times 10^{-8}$ & $3.4994 \times 10^{-8}$ \\
0.3 & 0.091382 & 0.091382 & 0.091383 & $9.1185 \times 10^{-7}$ & $9.1185 \times 10^{-7}$ \\
0.4 & 0.16445 & 0.16445 & 0.16446 & $9.3270 \times 10^{-6}$ & $9.3270 \times 10^{-6}$ \\
0.5 & 0.26111 & 0.26111 & 0.26117 & $5.7370 \times 10^{-5}$ & $5.7370 \times 10^{-5}$ \\
\hline
\end{tabular}

Figure 1, represents the behavior of the 4-term approximate solutions by NDM Caputo, NDM Caputo-Fabrizio and exact solution to some different values of $\alpha$. Figure 2, represents the comparison of the exact solution and 4-term approximate solutions by NDM Caputo at $\alpha=2$ and NDM Caputo-Fabrizio at $\alpha=1$. Table 1 , represents the numerical comparison between the 4-term approximate solutions by 

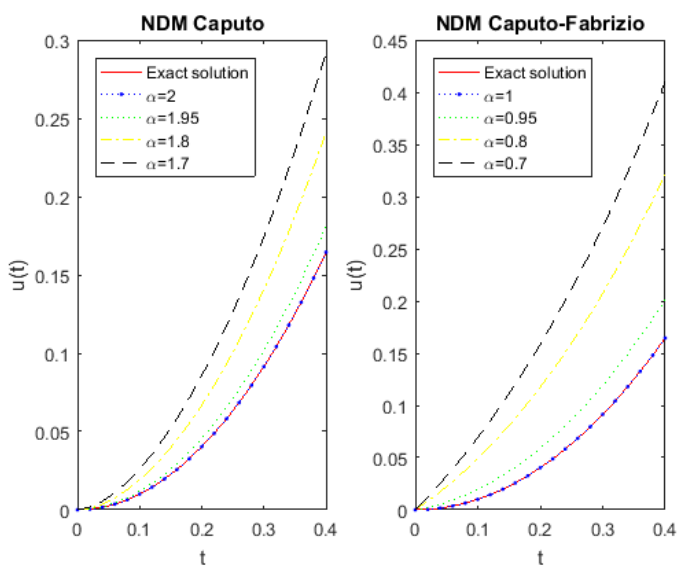

Fig. 1. The behavior of the exact solution and 4-term approximate solutions by NDM Caputo and NDM Caputo-Fabrizio for different values of $\alpha$

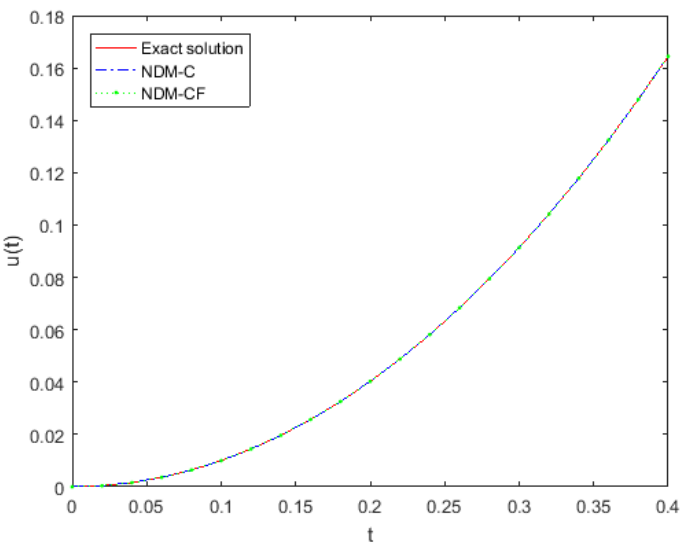

Fig. 2. Comparison of the exact solution and 4-term approximate solutions by NDM Caputo at $\alpha=2$ and NDM Caputo-Fabrizio at $\alpha=1$

NDM Caputo at $\alpha=2$ and NDM Caputo-Fabrizio at $\alpha=1$ and exact solution for differents values of $t$. Consequently the approximate solutions are rabidly convergence as the exact solution. In this paper, we only apply four terms to approximate the solutions, if we apply more terms of the approximate solutions, the accuracy of the approximate solutions will be greatly improved.

\section{Conclusions}

In this paper, the natural decomposition method (NDM) is used to solve the fractional Bratu's initial value problems in the Caputo and Caputo-Fabrizio sense. In order to illustrate the accuracy and effectiveness of the proposed method, it is 
applied to a numerical example. The approximate solutions obtained by this method are compared with the exact solutions. The numerical results obtained are in an excellent agreement with the exact solutions, therefore, we can conclude that the NDM is practically well suited for solving a wide range of fractional differential equations.

\section{References}

[1] Syam, M.I., \& Hamdan, A. (2006). An efficient method for solving Bratu equations. Appl. Math. Comput., 176(2), 704-713.

[2] Buckmire, R. (2004). Application of a Mickens finite-difference scheme to the cylindrical Bratu-Gelfand problem. Numer. Methods Partial Differential Equations, 20(3), 327-337.

[3] Jafari, H., \& Tajadodi, H. (2016). Electro-spunorganic nanofibers elaboration process investigations using BPs operational matrices. Iranian Journal of Mathematical Chemistry, 7(1), 19-27.

[4] Wazwaz, A.M. (2005). Adomian decomposition method for a reliable treatment of the Bratu-type equations. Appl. Math. Comput., 166, 652-663.

[5] Batiha, B. (2010). Numerial solution of Bratu-type equations by the variational iteration method. Hacet. J. Math. Stat., 39(1), 23-29.

[6] Feng, X., He, Y., \& Meng, J. (2008). Application of homotopy perturbation method to the Bratu-type equations. Topol. Methods Nonlinear Anal., 31, 243-252.

[7] Hassan, H.N., \& Semary, M.S. (2013). Analytic approximate solution for the Bratu's problem by optimal homotopy analysis method. Commun. Numer. Anal., 2013, 1-14.

[8] Deniz, S., \& Bildik, N. (2018). Optimal perturbation iteration method for Bratu-type problems. Journal of King Saud University Science, 30(1), 91-99.

[9] Abbasbandy, S., Hashemia, M.S., \& Liu, C-S. (2011). The Lie-group shooting method for solving the Bratu equation. Commun. Nonlinear Sci. Numer. Simul., 16(11), 4238-4249.

[10] Khalouta, A., \& Kadem, A. (2019). A new numerical technique for solving Caputo time-fractional biological population equation. AIMS Mathematics, 4(5), 1307-1319.

[11] Khalouta, A., \& Kadem, A. (2019). A new representation of exact solutions for nonlinear time-fractional wave-like equations with variable coefficients. Nonlinear Dyn. Syst. Theory, 19(2), 319-330.

[12] Bildik, N., \& Deniz, S., (2020). New approximate solutions to the nonlinear Klein-Gordon equations using perturbation iteration techniques. Discrete Contin. Dyn. Syst. Ser. S, 13(3), 503-518.

[13] Bildik, N., \& Deniz, S., (2019). A new fractional analysis on the polluted lakes system. Chaos Solitons Fractals, 122, 17-24.

[14] Yavuz, M. (2018). Novel recursive approximation for fractional nonlinear equations within Caputo-Fabrizio operator. ITM Web of Conferences, 22, 01008.

[15] Yavuz, M., \& Özdemir, N. (2018). European vanilla option pricing model of fractional order without singular kernel. Fractal and Fractional, 2(1), 1-11.

[16] Yavuz, M., \& Özdemir, N. (2020). Analysis of an epidemic spreading model with exponential decay law. Mathematical Sciences \& Applications E-Notes, 8(1), 1-13.

[17] Yavuz, M., \& Özdemir, N. (2020). Comparing the new fractional derivative operators involving exponential and Mittag-Leffler kernel. Discrete Contin. Dyn. Syst. Ser. S, 13(3), 995-1006.

[18] Keten, A., Yavuz, M., \& Baleanu, D. (2019). Nonlocal Cauchy problem via a fractional operator involving power kernel in Banach spaces. Fractal and Fractional, 3(2), 27, 1-8. 
[19] Kilbas, A., Srivastava, H.M., \& Trujillo, J.J. (2006). Theory and Application of Fractional Differential Equations. Amsterdam: Elsevier.

[20] Caputo, M., \& Fabrizio, M. (2015). A new definition of fractional derivative without singular kernel. Progress in Fractional Differentiation and Applications, 1(2), 73-85.

[21] Losada, J., \& Nieto, J.J. (2015). Properties of a new fractional derivative without singular kernel. Progress in Fractional Differentiation and Applications, 1(2), 87-92.

[22] Belgacem, F.B.M., \& Silambarasan, R. (2012). Theory of natural transform. Mathematics in Engineering, Science and Aerospace, 3(1), 105-135.

[23] Khalouta, A., \& Kadem, A. (2019). New technique for finding exact solution of nonlinear time-fractional wave-like equation with variable coefficients. Proc. Inst. Math. Natl. Acad. Sci. Ukr. Math. Appl., 45(2), 167-180.

[24] Zhu, Y., Chang, Q., \& Wu, S. (2005). A new algorithm for calculating Adomian polynomials. Appl. Math. Comput., 169, 402-416.

[25] Khalouta, A., \& Kadem, A. (2020). Solution of the fractional Bratu-type equation via fractional residual power series method. Appear in: Tatra Mt. Math. Publ. 